\title{
Desenvolvimento motor e sucesso académico. Que relação em crianças e jovens?
}

\author{
João Paulo Saraiva \\ Universidade do Minho, Portugal \\ Luís Paulo Rodrigues \\ Instituto Politécnico de Viana do Castelo, Portugal
}

\begin{abstract}
Resumo
A adopção de uma perspectiva holística do desenvolvimento obriga-nos a equacionar a relação entre vários elementos do desenvolvimento pessoal. Entre os mais relevantes destacam-se (1) o sucesso no desenvolvimento motor das crianças e jovens, pela sua relação com a saúde e a definição de estilos de vida saudáveis; e (2) o sucesso ou realização académica, pela sua valorização social, pela importância na determinação da vida pessoal (carreira), e pela presença constante em todos os momentos que antecedem a vida adulta. O presente trabalho constitui uma revisão da literatura existente sobre as relações existentes entre os factores associados ao desenvolvimento motor - nomeadamente a aptidão física, morfológica, coordenativa e a actividade física - e o sucesso académico de crianças e jovens, revisitados numa perspectiva holística.
\end{abstract}

Palavras-chave

Sucesso académico; Desenvolvimento motor; Crianças; Adolescentes

\section{Introdução}

A expressão do poeta Juvenal "mente sã em corpo são" tem sido historicamente utilizada para avisar as sociedades das consequências 
nefastas para a saúde de um estilo de vida sedentário, bem como para expor os benefícios comuns de um desenvolvimento equilibrado. Aliás, o entendimento actual dos níveis óptimos de saúde dos indivíduos (Who, 1999) privilegia necessariamente esse entendimento holístico. Talvez por isso, a investigação acerca das relações e/ou efeitos dos estilos de vida activos no sucesso académico e desempenho escolar em crianças e jovens tem constituído uma preocupação actual e incontornável para o entendimento do processo de desenvolvimento do ser humano num contexto global.

Parece consensual a ideia de que o fomento da prática de exercício físico regular em tenras idades tem reflexos notórios na adopção de estilos de vida saudáveis que perduram no tempo. Na mesma medida, espera-se que o processo de desenvolvimento motor (DM) iniciado na infância tenha impacto futuro na qualidade de vida das populações, emergindo assim a escola como cenário ideal para a sua estruturação. Para além dos benefícios atribuídos à prática regular de exercício físico, com reflexos evidentes ao nível da saúde, aptidão física, morfológica e coordenativa, o percurso de DM da criança poderá abranger outras facetas do desenvolvimento humano. Entre estas, o sucesso ou desempenho escolar, pela partilha geracional de um conjunto de aquisições sociais relevantes que se constituem como património cultural na sua vertente de construção do relacionamento e integração na sociedade, assume uma das suas expressões mais palpáveis, sobretudo em idades precoces.

Se é verdade que o nosso sistema educativo tem privilegiado uma perspectiva ampla, holística das necessidades educativas do jovem em desenvolvimento, não é menos verdade que o peso da maior relevância social de certas áreas de aprendizagem tem vindo a desequilibrar a balança no sentido do reforço dos tempos de aprendizagem das matérias ditas "nucleares" (e.g. matemática e língua materna). De facto, o sucesso escolar ou académico constitui hoje, para as famílias, uma fonte de preocupação fundamental no desenvolvimento de crianças e jovens, originando que desde muito cedo exista uma pressão social para a valorização extremada das notas e classificações escolares que podem dar acesso, no futuro, a formações superiores de maior relevância social e êxito profissional (veja-se o caso da Medicina e da Arquitectura, por exemplo). Este tipo de preocupações têm infelizmente recentrado as preocupações dos intervenientes no processo 
educativo nas velhas máximas do "ler, contar e escrever", mesmo que travestidas agora de um nível de dificuldade adequado aos tempos modernos. No outro extremo, e como consequência, as áreas das expressões, com a Educação Física à cabeça, tendem a ser relegadas na distribuição do tempo e da organização das escolas.

Nos antípodas desta realidade, a eventualidade de que o sucesso escolar possa estar correlacionado de forma geral com o êxito nos aspectos particularmente marcantes do DM na infância e que determinam os estilos de vida na adolescência e idade adulta tem ocupado recorrentemente os investigadores desenvolvimentalistas ao longo dos tempos, e de forma particularmente intensa na última década. Estes aspectos ou variáveis marcadoras do DM da criança e jovem são: Aptidão Física (ApF), Actividade Física (AF), Aptidão Coordenativa (ApC) e Aptidão Morfológica (ApM).

A ApF refere-se ao nível evidenciado pelas crianças e jovens em provas ou testes de força, velocidade, resistência aeróbia, agilidade e flexibilidade. Representa elemento fundamental para o sucesso motor, propiciando êxito na actividade e criando expectativas de manutenção futura de estilos de vida activos (Andersen, Hasseltrom, Gronfeldt, Hansen, \& Karsten, 2004). O envolvimento em práticas de AF tem sido descrito como um dos mediadores mais importantes ao sucesso motor nas primeiras idades, sendo ainda factor relevante na determinação epidemiológica da saúde das populações (Blair \& Church, 2004). A ApC expressa a qualidade, a coordenação do movimento e tem vindo a evidenciar-se na literatura dos últimos anos devido à sugestão de que os níveis de ApC poderão influenciar decisivamente a forma como as crianças se envolvem e motivam em práticas motoras desafiantes. E, por último, a ApM é entendida como as características morfológicas associadas ao nível de prontidão motora e robustez músculoesquelética do indivíduo. Tem sido observada através de indicadores dimensionais simples (estatura, peso, perímetros musculares e pregas adiposas) e complexos (por exemplo, o índice de massa corporal).

Por outro lado, referimo-nos neste texto ao Sucesso Académico (SA), tradução para o termo inglês Academic Achievement normalmente utilizado pelos autores dos artigos aqui revistos e que se refere às condições objectivas que permitem avaliar o desempenho dos alunos (notas), ou às particularidades também objectivas que referenciam condições de êxito nas 
aprendizagens escolares (concentração, memória, etc.) e que por vezes são também referenciadas como características de desempenho cognitivo.

Numa meta-análise dos estudos publicados até 2002, e que examinaram a relação entre a AF e o SA ou o desempenho cognitivo, Sibley e Etnier (2003) concluíram que: (1) estes factores se associavam positiva e significativamente em crianças; (2) o efeito era independente da intensidade da AF; e (3) a magnitude do efeito (0.40) se revelava maior em crianças pertencentes ao $1^{\circ}$ ciclo do ensino básico. Taras (2005), numa revisão de catorze estudos que trataram especificamente a relação entre a AF e várias medidas do SA em crianças e jovens em idade escolar (5-18 anos), divide as suas conclusões pela existência de uma associação significativa mas fraca entre a AF e o SA, ou pela inexistência de qualquer correlação, mas sobretudo sugere a necessidade de maior informação nesta matéria.

Burton e VanHeest publicaram em 2007 um artigo de revisão onde abordam o papel da AF na atenuação do fosso académico entre etnias nos EUA. Nas palavras dos autores, os estudos sugerem que a AF tem sido reconhecida como benéfica para o desempenho cognitivo das crianças. Complementarmente, a AF é também identificada como influência positiva na redução do excesso de peso e obesidade que atinge sobretudo as crianças dos grupos étnicos minoritários (negros e hispânicos). Mais recentemente, Tomporowski, Davis, Miller, e Naglieri (2008), numa revisão que ampliou a de 2003 de Sibley e Etnier, concluem que: (1) crianças fisicamente mais aptas são mais rápidas no desempenho de tarefas cognitivas e demonstram padrões de actividade neurofisiológica indicativos de maior mobilização cerebral; (2) apesar de crianças sujeitas a maior AF apresentarem geralmente melhores índices de SA, os resultados não são totalmente esclarecedores desta relação devido às limitações e possíveis enviezamentos dos estudos; (3) em todo o caso, é claro que o tempo dispendido em aulas de Educação Física escolar não é deletério do progresso académico das crianças.

O que nos propomos neste artigo é rever os estudos publicados nesta temática, ampliando e completando as revisões já efectuadas em língua inglesa com os resultados encontrados na última década. O objectivo fundamental será o de perceber as conclusões da investigação acerca das relações que um DM bem sucedido pode ter na determinação do sucesso académico global das crianças e jovens. Será que ter êxito nas componentes 
associadas ao DM se coaduna com o desempenho escolar geral? Ou, pelo contrário, indicará a investigação que os domínios das aprendizagens cognitivas e das aprendizagens motoras são independentes ou até mesmo antagónicos, como parece decorrer da visão sociocultural que procura atribuir ao tempo de actividade motora um papel subalterno (quiçá dispensável) no processo educativo?

\section{Metodologia}

$\mathrm{Na}$ pesquisa dos estudos publicados recorremos à Biblioteca do Conhecimento Online (B-on), base bibliográfica online que junta diversos recursos de pesquisa (PubMed, Science Direct, Web of Science, Scielo, Academic Search, SpringerLink, etc.). Os critérios de procura seleccionados permitiram-nos consultar os trabalhos publicados na última década (2000 a 2009) através do cruzamento de palavras-chave especificamente relacionadas com o objectivo da revisão (Academic Achievement, Cognitive Skills, Motor Skills, Physical Activity, Physical Fitness, Obesity, Growth). Outros estudos anteriores a esta década foram também consultados através da referenciação feita nos artigos consultados. Foram incluídos nesta revisão sistemática todos os estudos cujo delineamento procurava associações ou efeitos entre o SA e as variáveis de interesse para o DM (aptidão física, actividade física, aptidão coordenativa, aptidão morfológica), resultando num total de 21 artigos.

No conjunto dos trabalhos revistos, diferentes variáveis são definidas como marcadoras do desempenho académico. As mais usadas são as classificações atribuídas aos alunos pelos professores e os testes estandardizados nas diversas áreas do currículo; no entanto, também foram utilizadas outras variáveis associadas ao desempenho cognitivo (capacidade de concentração, aprendizagem, memorização, comportamento em sala de aula, auto-estima, etc.).

Relativamente às formas de inquirição utilizadas pelos diferentes autores para esclarecer esta questão, encontramos sobretudo estudos de natureza quasi-experimental ou de intervenção, e estudos correlacionais. Os primeiros caracterizam-se pelo controlo e manipulação deliberada das condições de prática da AF (usualmente pela introdução de programas de educação motora e actividade física) e a observação do seu impacto sobre o 
SA. Os segundos procuram inferir sobre a relação entre as variáveis associadas ao desempenho motor e AF, e o SA. Um e outro tipo recorrem a delineamentos longitudinais e transversais de estudo, sendo que neste último caso se torna mais difícil controlar outras potenciais influências, nomeadamente o estauto sócio-económico da amostra envolvida (Willms, 2003).

É seguindo esta mesma perspectiva que estruturamos a apresentação desta revisão sistemática em: (1) estudos sobre o impacto, no SA, de programas de intervenção motora e de actividade física; e (2) estudos sobre a associação do SA com a AF, a ApF, a ApM e a ApC de crianças e jovens em idade escolar. Para cada um dos estudos procurou-se descrever o ano, 0 local/país, as características da amostra, o delineamento experimental e os resultados obtidos. Para ajudar o leitor na comparação, foi elaborada uma tabela-síntese de todos os estudos revistos. Os resultados da revisão foram organizados pelo ano da sua publicação, de forma a que o leitor possa formular uma ideia acerca da crescente preocupação dos investigadores nesta matéria.

\section{Revisão da Literatura}

\section{Estudos de intervenção (longitudinais e quasi-experimentais)}

Num estudo que pretendia observar os efeitos sobre a saúde de intervenções variadas de educação física no currículo escolar, 500 crianças australianas com 10 anos de idade participaram durante dois anos num de dois programas diários suplementares (de melhoria da aptidão física, ou das habilidades motoras), ou apenas nas aulas de educação física. Dwyer, Coonan, Leitch, Hetzel, e Baghurst (1983) concluíram que: (1) os alunos participantes num dos dois primeiros programas tiveram benefícios evidentes nos resultados associados com a saúde; (2) os resultados escolares (testes de aritmética e leitura) não foram afectados negativamente pelas alterações curriculares introduzidas a favor da actividade física diária, mesmo que isso tivesse significado uma redução diária de 45 a 60 minutos no tempo de leccionação dessas matérias curriculares.

Shephard et al. (1984), no já famoso estudo de Trois Riviéres (Québec, Canadá), seguiram 546 crianças do ensino primário do primeiro ao sexto ano de escolaridade. Estas foram divididas em dois grupos - experimental e de controlo - , sendo que o primeiro cumpriu um programa de 300 minutos 
adicionais de educação física por semana, enquanto o segundo grupo cumpriu apenas com o programa escolar em vigor de $40 \mathrm{~min} / \mathrm{semana}$. Os resultados dos exames revelaram que as crianças que integravam o grupo de intervenção obtiveram melhores classificações em Matemática, mas um rendimento inferior em Inglês (segunda língua oficial).

Em Israel, Raviv, Reches e Hecht (1994) implementaram durante um ano um programa educativo voltado para o movimento corporal com um grupo experimental de 92 crianças do Jardim de Infância e 266 do $1^{\circ}$ ano de escolaridade, e verificaram que estes alunos obtiveram melhores resultados ao nível da leitura e da aritmética do que o grupo de controlo.

\section{Quadro 1 - Estudos de intervenção (quasi-experimentais e} longitudinais)

\begin{tabular}{|c|c|c|c|c|}
\hline Autor(es) & Pais & Amostra & Intervençāo & Conclusōes \\
\hline $\begin{array}{l}\text { Dwyer, Coonan, } \\
\text { Leitch, Hetzel, \& } \\
\text { Baghurst (1983) }\end{array}$ & AUS & $\begin{array}{l}500 \text { alunos com } \\
10 \text { anos de } \\
\text { idade }\end{array}$ & $\begin{array}{l}\text { Programa diário de aptidåo } \\
\text { fisica ou desenvolvimento das } \\
\text { habilidades motoras, durante } \\
\text { dols anos. }\end{array}$ & $\begin{array}{l}\text { Resultados escolares (Aritmética e } \\
\text { Leitura) nấo foram afectados } \\
\text { negativamente pelas alteraçbes } \\
\text { curriculares introduzidas a favor dos } \\
\text { programas de actividades motoras. } \\
\text { Beneficios evidentes ao nivel da } \\
\text { saúde. }\end{array}$ \\
\hline $\begin{array}{l}\text { Shephard et al. } \\
\text { (1984) }\end{array}$ & CAN & $\begin{array}{l}546 \text { alunos } \\
\text { seguidos desde } \\
01 .^{\circ} \text { ao } 6 . .^{\circ} \text { ano. }\end{array}$ & $\begin{array}{l}\text { Programa de } 300 \\
\text { min/semana, durante seis } \\
\text { anos, de Educaçăo Fisica } \\
\text { orientada. }\end{array}$ & $\begin{array}{l}\text { Melhores classificap̧os no exame } \\
\text { de Matemática, mas năo no de } \\
\text { Inglés. Melhoria nas notas } \\
\text { escolares. Melhoria nos testes } \\
\text { WISC. }\end{array}$ \\
\hline $\begin{array}{l}\text { Raviv, Reches, \& } \\
\text { Hecth (1994) }\end{array}$ & ISR & $\begin{array}{l}358 \text { alunos do } \\
\text { Jardim de } \\
\text { Infáncia e 1.॰ } \\
\text { ano de } \\
\text { escolaridade: }\end{array}$ & $\begin{array}{l}\text { Programa educativo de } \\
\text { movimento corporal durante } \\
\text { um ano. }\end{array}$ & Melhorias na leitura $e$ aritmética. \\
\hline Sallis et al. (1999) & EUA & $\begin{array}{l}655 \text { alunos dos } \\
5 .^{\circ} \text { e } 6 .^{\circ} \text { anos de } \\
\text { escolaridade; }\end{array}$ & $\begin{array}{l}\text { Programa adicional de } \\
\text { educą̧äo física relacionada } \\
\text { com a saúde (SPARK) } \\
\text { durante dois anos. }\end{array}$ & $\begin{array}{l}\text { Efeitos positivos na generalidade } \\
\text { das componentes do Metropolitan } \\
\text { Achievement Test. }\end{array}$ \\
\hline $\begin{array}{l}\text { Coe, Pivarnik, } \\
\text { Womack, Reeves, } \\
\text { \& Malina (2006) }\end{array}$ & EUA & $\begin{array}{l}214 \text { alunos do } \\
6 .{ }^{\circ} \text { ano de } \\
\text { escolaridade; }\end{array}$ & $\begin{array}{l}\text { Programa curricular de } \\
\text { Educaçäo Fisica durante um } \\
\text { ano. } \\
\text { Avaliaçäo dos niveis de AF } \\
\text { diários. }\end{array}$ & $\begin{array}{l}\text { A participação em aulas de } \\
\text { Educaçấo Fisica nấo demonstrou } \\
\text { nenhuma associaçấo particular com } \\
\text { o SA. } \\
\text { Alunos que apresentaram niveis } \\
\text { diários de AF vigorosa tiveram } \\
\text { melhores notas curriculares. }\end{array}$ \\
\hline $\begin{array}{l}\text { Ahamed, } \\
\text { Macdonald, Feed, } \\
\text { Naylor, Lui- } \\
\text { Ambrose, \& } \\
\text { McKay (2007) }\end{array}$ & CAN & $\begin{array}{l}287 \text { alunos dos } \\
4 .^{\circ} \text { e } 5 .^{\circ} \text { anos de } \\
\text { escolaridade; }\end{array}$ & $\begin{array}{l}\text { Programa de promoçấa } \\
\text { escolar da AF (AS!BC). } 47 \\
\text { minutos adicionais durante } 16 \\
\text { meses. }\end{array}$ & $\begin{array}{l}\text { Verificou-se uma tendência para o } \\
\text { reforço dos resultados no testes } \\
\text { CAT-3 no grupo de intervençắo. }\end{array}$ \\
\hline $\begin{array}{l}\text { Carison et al. } \\
\text { (2008) }\end{array}$ & EUA & $\begin{array}{l}5316 \text { alunos } \\
\text { seguidos do } \\
\text { Jardim de } \\
\text { Infância ao } 5^{\circ} \\
\text { ano. }\end{array}$ & $\begin{array}{l}\text { Programas de Educaçăo } \\
\text { Fisica que variavam de } 70 \\
\text { min/semana até } 300 \\
\text { min/semana durante um ano. }\end{array}$ & $\begin{array}{l}\text { Associaçäo positiva, observada } \\
\text { apenas em raparigas, entre a } \\
\text { frequência nas aulas de EF e os } \\
\text { resultados académicos ao nivel da } \\
\text { Leitura e da Matemática; }\end{array}$ \\
\hline
\end{tabular}


200 João Paulo Saraiva \& Luís Paulo Rodrigues

Sallis et al. (1999) testaram o efeito de um programa de educação física relacionado com a saúde (SPARK) no desempenho escolar, avaliado pelo Metropolitan Achievement Test (MAT), de 759 crianças norte-americanas do $5 .^{\circ}$ e $6 .^{\circ}$ anos de escolaridade. Três grupos foram sujeitos a diferentes condições: sessões com especialistas, sessões orientadas pelo professor da classe, e sem intervenção. Ao fim de dois anos, o grupo com sessões orientadas pelo professor da classe obteve resultados superiores ao grupo controle em três das quatro componentes dos testes que constituem o MAT; e o grupo que trabalhou com o especialista teve resultados superiores num dos testes e inferiores noutro. Os autores concluem que, apesar de os dois grupos que beneficiaram do programa SPARK terem ocupado o dobro do tempo curricular em aulas de educação física (em detrimento das outras matérias), este facto não interferiu negativamente no SA, sendo mesmo sugerido que na globalidade os efeitos foram positivos.

Por sua vez, Coe, Pivarnik, Womack, Reeves, e Malina (2006), ao estudarem o efeito da participação em sessões de EF e dos níveis de AF fora da escola (3DPAR) sobre o SA (notas curriculares e testes padronizados) de 214 alunos de ambos os géneros do $6 .^{\circ}$ de escolaridade, concluíram que os alunos que se envolviam em actividades físicas vigorosas obtiveram um desempenho académico (notas curriculares) significativamente superior aos demais. No Canadá, e com propósito idêntico, 287 estudantes de ambos os géneros dos $4 .^{\circ}$ e $5 .^{\circ}$ anos foram divididos em dois grupos (Ahamed et al., 2007). O grupo experimental teve 47 minutos adicionais de actividades físicas por semana relativamente ao grupo de controlo. Apesar da correspondente diminuição do tempo de ensino das matérias curriculares convencionais, a performance académica do grupo experimental não sofreu alterações, revelando mesmo uma tendência para melhorar.

Mais recentemente, Carlson et al. (2008) examinaram a associação entre o tempo dispendido em EF e o SA no contexto de um estudo longitudinal com 5.316 estudantes americanos do Jardim de Infância ao $5 .^{\circ}$ ano de escolaridade, tendo observado que as raparigas expostas a maiores períodos de frequência em EF (70-300 min/semana) revelaram ligeira melhoria académica ao nível da Matemática e da Leitura relativamente àquelas que estiveram expostas a períodos inferiores (0-35 min/semana). Não foi observada qualquer associação nos rapazes. Os autores concluem que os 
resultados do estudo suportam a teoria de que o tempo gasto na prática de EF não afecta negativamente o SA e que pode, inclusive, em alguns casos, favorecer modestamente o seu desempenho.

\section{Estudos de associação (correlacionais e transversais)}

Pate, Heath, Dowda, e Trost (1996), num estudo com 11.631 adolescentes americanos de ambos os géneros (dos 12 aos 18 anos de idade) que pretendeu investigar a influência dos níveis de AF sobre a probabilidade de adopção de comportamentos de risco para a saúde (CRS), verificaram que adolescentes com pouco ou nenhum envolvimento em AF demonstravam mais comportamentos negativos (tabagismo, consumo de drogas, maus hábitos alimentares, sedentarismo) e uma baixa autopercepção do SA.

Mo-Suwan, Lebel, Puetpaiboon, e Junjana (1999) estudaram a relação entre o Índice de Massa Corporal (IMC) e a média aritmética das matérias curriculares (SA) de 1.794 crianças tailandesas de ambos os géneros do $3^{\circ}$ ao $9^{\circ}$ ano de escolaridade. Nos alunos do $3^{\circ}$ ao $6^{\circ}$ ano não foram encontradas associações entre o SA e qualquer das categorias de ApM definidas tendo por base o IMC de cada aluno (baixo peso, peso normal, sobrepeso, obesidade). Contudo, entre as crianças do $7^{\circ}$ ao $9^{\circ}$ ano de escolaridade, as crianças com excesso de peso obtiveram notas médias inferiores.

Dwyer, Sallis, Blizzard, Lazarus, e Dean (2001), num delineamento transversal com 9.000 alunos australianos de ambos os géneros dos 7 aos 15 anos de idade, observaram entre os 9 e os 12 anos uma associação positiva entre a performance académica (SA) e o nível de actividade física das crianças. Segundo os autores, os valores de correlação foram baixos mas estatisticamente significativos, sugerindo que a AF contribuiu efectivamente para o SA dos participantes. 
202 João Paulo Saraiva \& Luís Paulo Rodrigues

Quadro 2 - Estudos de associação (correlacionais e transversais)

\begin{tabular}{|c|c|c|c|c|}
\hline Autores(es) & Pais & Amostra & Variáveis & Conclusōes \\
\hline $\begin{array}{l}\text { Pate, Heath, } \\
\text { Dowda, \& Trost } \\
\text { (1996) }\end{array}$ & EUA & $\begin{array}{l}11 \text { e31 } \\
\text { adolescentes dos } \\
12 \text { aos } 18 \text { anos de } \\
\text { idade. }\end{array}$ & $\begin{array}{l}\text { Actividade fisica, } \\
\text { Comportamentos de risco } \\
\text { para a saúde, e auto- } \\
\text { percepçáo do Sucesso } \\
\text { Académico (Youth Fisk } \\
\text { Behavior Survey). }\end{array}$ & $\begin{array}{l}\text { Pouco ou nenhum } \\
\text { envolvimento em AF foil } \\
\text { associado com a evidêncla de } \\
\text { maior número de } \\
\text { comportamentos de riscos } \\
\text { para a saúde, e com uma } \\
\text { baixa percepçä́o do seu SA. }\end{array}$ \\
\hline $\begin{array}{l}\text { Mo-Suwan, Lebel, } \\
\text { Puetpaiboon, \& } \\
\text { Junjana (1999) }\end{array}$ & TAI & $\begin{array}{l}1794 \text { alunos do } \\
3 .^{\circ} \text { ao } 9 .^{\circ} \text { ano de } \\
\text { escolaridade. }\end{array}$ & $\begin{array}{l}\text { Aptidäo morfológica } \\
\text { (peso, altura e IMC) eo } \\
\text { Sucesso Académico } \\
\text { (notas escolares). }\end{array}$ & $\begin{array}{l}\text { Ter excesso de peso durante a } \\
\text { adolescencia encontra-se } \\
\text { associado com pior } \\
\text { desempenho escolar. }\end{array}$ \\
\hline $\begin{array}{l}\text { Dwyer, Sallis, } \\
\text { Blizzard, Lazarus, } \\
\text { \&. Dean (2001) }\end{array}$ & AUS & $\begin{array}{l}7961 \text { alunos dos } \\
7 \text { aos } 15 \text { anos de } \\
\text { idade. }\end{array}$ & $\begin{array}{l}\text { Desempenho escolar } \\
\text { (notas escolares), } \\
\text { Actividade Fisica } \\
\text { (questionário); Aptidäo } \\
\text { Fisica (PWC,70). }\end{array}$ & $\begin{array}{l}\text { A AF contribuiu para o reforepo } \\
\text { do SA dos participantes no } \\
\text { estudo. }\end{array}$ \\
\hline Kim et al. (2003) & KOR & $\begin{array}{l}6463 \text { alunos do } \\
5 .^{\circ}, 88^{\circ} \text { e } 111^{\circ} \text { ano } \\
\text { de escolaridade. }\end{array}$ & $\begin{array}{l}\text { Aptidäo Fisica (bateria de } \\
\text { testes escolares). } \\
\text { Sucesso Académioo } \\
\text { (notas escolares). }\end{array}$ & $\begin{array}{l}\text { Associaçäo positiva mas de } \\
\text { pequena magnilude, entre a } \\
\text { ApF e o SA. }\end{array}$ \\
\hline \multirow[t]{2}{*}{ Grissom (2005) } & \multirow[t]{2}{*}{ EUA } & \multirow{2}{*}{$\begin{array}{l}884715 \text { alunos } \\
\text { dos } 5 .^{\circ}, 7 .^{\circ} \text { e } 9 .^{\circ} \\
\text { anos de } \\
\text { escolaridade. }\end{array}$} & \multirow{2}{*}{$\begin{array}{l}\text { Aptidalo Fisica } \\
\text { (FITNESSGRAM), Leitura } \\
\text { e Matemática (Stanford } \\
\text { Achievement Test } g^{\prime \prime} \text { ed.). }\end{array}$} & $\begin{array}{l}\text { Relaçäo positiva e consistente } \\
\text { entre ApF e o SA. }\end{array}$ \\
\hline & & & & $\begin{array}{l}\text { Raparigas com niveis mais } \\
\text { elevados de ApF, e alunos } \\
\text { com estatuto sócio-económico } \\
\text { mais alto obtiveram } \\
\text { tendencialmente melhores } \\
\text { notas no SAT. }\end{array}$ \\
\hline $\begin{array}{l}\text { Hillman, Castelli, } \\
\text { \&. Buck (2005) }\end{array}$ & EUA & $\begin{array}{l}24 \text { crianças ( } 9,6 \\
\text { anos) } \text { e } 27 \text { adultos } \\
\text { (19,3 anos). }\end{array}$ & $\begin{array}{l}\text { Aptidäo Fisica } \\
\text { (FITNESSGRAM) e } \\
\text { funçăo cognitiva } \\
\text { (respostas } \\
\text { comportamentais e } \\
\text { neuroelétrica a um } \\
\text { estímulo). }\end{array}$ & $\begin{array}{l}\text { ApF positivamente associada } \\
\text { com a actividade } \\
\text { neuroeléctrica responsável } \\
\text { pela atençâo, memória e } \\
\text { velocidade de resposta em } \\
\text { crianças; com a funçăo } \\
\text { cognitiva em pré. } \\
\text { adolescentes; }\end{array}$ \\
\hline $\begin{array}{l}\text { Wassenberg et al. } \\
(2005)\end{array}$ & $\mathrm{HOL}$ & $\begin{array}{l}378 \text { crianças com } \\
5 \text { e } 6 \text { anos de } \\
\text { idade. }\end{array}$ & $\begin{array}{l}\text { Desempenho Motor, } \\
\text { Integraçäo Visuo-motora e } \\
\text { Memória de Trabalho. }\end{array}$ & $\begin{array}{l}\text { Relap̧bes positivas entre } \\
\text { desempenho motor, } \\
\text { integraçäo visuo-motora e } \\
\text { memória de trabalho; e entre } \\
\text { aspectos quantitativos do } \\
\text { desempenho motor e a } \\
\text { fluência verbal. }\end{array}$ \\
\hline
\end{tabular}




\begin{tabular}{|c|c|c|c|c|}
\hline $\begin{array}{l}\text { Yu, Chang. } \\
\text { Cheng, Sun, \&. } \\
\text { Hau (2006) }\end{array}$ & $\mathrm{CHI}$ & $\begin{array}{l}333 \text { crianças dos } \\
8 \text { aos } 12 \text { anos de } \\
\text { idade. }\end{array}$ & $\begin{array}{l}\text { Actividade Fisica } \\
\text { (questionário PAQ-C), SA } \\
\text { (notas escolares). }\end{array}$ & $\begin{array}{l}\text { Ausência de associaçăo } \\
\text { significativa entre os níveis de } \\
\text { Af e os resultados escolares }\end{array}$ \\
\hline $\begin{array}{l}\text { Nelson \& Gordon- } \\
\text { Larsen (2006) }\end{array}$ & EUA & $\begin{array}{l}11957 \text { crianças } \\
\text { dos } 7 \text { aos } 12 \text { anos } \\
\text { de idade. }\end{array}$ & $\begin{array}{l}\text { Actividade Fisica } \\
\text { (questionário), e Sucesso } \\
\text { Académico (notas } \\
\text { escolares). }\end{array}$ & $\begin{array}{l}\text { Adolescentes fisicamente mais } \\
\text { activos revelaram maior } \\
\text { probabilidade de atingir } \\
\text { melhores resultados } \\
\text { académicos. }\end{array}$ \\
\hline $\begin{array}{l}\text { Sigtúsdóttir, } \\
\text { Kristjánsson, \& } \\
\text { Allegrante (2007) }\end{array}$ & ISL & $\begin{array}{l}5810 \text { alunos com } \\
14 \text { e } 15 \text { anos de } \\
\text { idade. }\end{array}$ & $\begin{array}{l}\text { Actividade Fisica } \\
\text { (questionário) e Sucesso } \\
\text { Académico (notas } \\
\text { escolares). }\end{array}$ & $\begin{array}{l}\text { Pequena mas significante } \\
\text { associaçä́o positiva entre AF e } \\
\text { as notas escolares. }\end{array}$ \\
\hline \multirow[t]{2}{*}{$\begin{array}{l}\text { Cottrell, Northrup, } \\
\& \text { Wittberg (2007) }\end{array}$} & \multirow[t]{2}{*}{ EUA } & \multirow[t]{2}{*}{$\begin{array}{l}968 \text { alunos do } 5^{\circ} \\
\text { ano de } \\
\text { escolaridade. }\end{array}$} & \multirow[t]{2}{*}{$\begin{array}{l}\text { Aptidāo Mortologica } \\
\text { (IMC), Aptidäo Fisica } \\
\text { (FITNESSGRAM) e } \\
\text { Sucesso Académico } \\
\text { (West Virginia Educational } \\
\text { Standards Test). }\end{array}$} & $\begin{array}{l}\text { Em crianças consideradas } \\
\text { obesas foi encontrada uma } \\
\text { associaçäo inversa } e \\
\text { significativa com os resultados } \\
\text { no WVEST, excepto em } \\
\text { Estudos Sociais. }\end{array}$ \\
\hline & & & & $\begin{array}{l}\text { Crianças com menores niveis } \\
\text { de ApF obtiveram valores } \\
\text { mais baixos no WVEST. }\end{array}$ \\
\hline $\begin{array}{l}\text { Martin \& Chalmers } \\
(2007)\end{array}$ & EUA & $\begin{array}{l}5847 \text { alunos do } \\
3 .^{\circ}, 4 .^{\circ}, 6 .^{\circ} \text { e } 8 .^{\circ} \\
\text { ano de } \\
\text { escolaridade. }\end{array}$ & $\begin{array}{l}\text { Aptidāo Fisica } \\
\text { (President's Challenge } \\
\text { Youth Fitness Test) e } \\
\text { Sucesso Académico (lowa } \\
\text { Test of Basic Skills). }\end{array}$ & $\begin{array}{l}\text { A associaçăo entre a ApF e } 0 \\
\text { SA foi positiva e } \\
\text { estatisticamente significativa } \\
\text { mas de baixo valor }\end{array}$ \\
\hline $\begin{array}{l}\text { Castelli, Hillman, } \\
\text { Buck, \& Erwin } \\
\text { (2007) }\end{array}$ & EUA & $\begin{array}{l}582 \text { alunos do } 3 .^{\circ} \\
\text { e } 5 .^{\circ} \text { ano de } \\
\text { escolaridade. }\end{array}$ & $\begin{array}{l}\text { Aptidāo Fisica (PACER), } \\
\text { Aptidăo Mortológica (IMC) } \\
\text { e Sucesso Académico } \\
\text { (Wiinois Standard } \\
\text { Achievement Test). }\end{array}$ & $\begin{array}{l}\text { Correlaçăo positiva entre a } \\
\text { ApF e o SA, e uma correlaçä́o } \\
\text { negativa entre a ApM e o SA } \\
\text { dos participantes; }\end{array}$ \\
\hline \multirow[t]{2}{*}{$\begin{array}{l}\text { Chomitz et al. } \\
(2009)\end{array}$} & \multirow[t]{2}{*}{ EUA } & \multirow{2}{*}{$\begin{array}{l}1847 \text { alunos dos } \\
4 .^{\circ}, 6 .^{\circ}, 7 .^{\circ} \text { e } 8 .^{\circ} \\
\text { anos de } \\
\text { escolaridade. }\end{array}$} & \multirow{2}{*}{$\begin{array}{l}\text { Aptidäo Fisica } \\
\text { (FITNESSGRAM) e } \\
\text { Sucesso Académico } \\
\text { (Massachusetts } \\
\text { Comprehensive } \\
\text { Assesment System). }\end{array}$} & $\begin{array}{l}\text { Associaçäo significativa entre } \\
\text { ApF e SA. }\end{array}$ \\
\hline & & & & $\begin{array}{l}\text { A probabilidade de sucesso } \\
\text { das crianças nos dois testes } \\
\text { do MCAS aumentou à medida } \\
\text { que aumentou a quantidade } \\
\text { de componentes positivas nos } \\
\text { testes de ApF. }\end{array}$ \\
\hline
\end{tabular}

Kim et al. (2003), ao estudarem a associação entre os hábitos alimentares (questionário sobre a frequência e a qualidade das refeições), a $\mathrm{ApF}$ (resultados de testes anuais realizados pelas escolas), o estatuto sócioeconómico (nível de instrução dos encarregados de educação) e o SA (média das classificações obtidas nas disciplinas curriculares) de 6.463 alunos coreanos de ambos os géneros do $5 .^{\circ}, 8^{\circ}$ e $11 .^{\circ}$ anos de escolaridade, observaram uma fraca mas positiva associação entre a ApF e o SA.

Num grande estudo que envolveu 884.715 crianças californianas dos 5..$^{\circ} 7^{\circ}$ e $9 .^{\circ}$ anos de escolaridade, Grissom (2005) observou relações positivas lineares entre a aptidão física (FITNESSGRAM) global e os 
resultados ao nível da Leitura e da Matemática (Stanford Achievement Test 9th edition). Esta relação foi mais forte entre as raparigas com níveis mais elevados de condição física. No mesmo ano, Hillman, Castelli e Buck (2005), investigando sobre a relação entre a idade, a ApF e a função cognitiva de 24 crianças de ambos os géneros (média de idades de 9,6 anos) e a posterior comparação destes dados com 27 adultos (média de idades de 19,3 anos), verificaram que a ApF esteve positivamente associada com a actividade neuroeléctrica responsável pela atenção, memória e velocidade de resposta em crianças, bem como com a função cognitiva em pré-adolescentes, demonstrando implicações para o aumento da função cognitiva em adultos. $\mathrm{Na}$ relação entre o desempenho motor e o cognitivo, numa amostra de 378 crianças holandesas de 5 e 6 anos de ambos os géneros, Wassenberg et al. (2005) não encontraram relação nos resultados globais, quando controlados para a atenção. No entanto, os autores descrevem relações positivas entre alguns aspectos específicos das componentes de cognição avaliadas, nomeadamente entre o desempenho motor, a integração visuo-motora, a memória de trabalho (working memory) e a fluência verbal.

Em 2006, Nelson e Gordon-Larsen, num estudo sobre as relações entre a AF (questionário sobre a frequência), os padrões de comportamento sedentário (visionamento de TV, videojogos e jogos de computador) e uma variedade de comportamentos de risco (tabagismos, alcoolismo, DST, consumo de drogas e delinquência) de 11.957 crianças de ambos os géneros dos 7 aos 12 anos de idade, observaram que os adolescentes fisicamente mais activos revelaram maior probabilidade de atingir melhores resultados académicos, enquanto os comportamentos sendentários foram considerados como eventuais potenciadores dos comportamentos de risco. Resultados diferentes foram observados por Yu, Chan, Cheng, Sung, e Hau (2006) na China. Num estudo sobre as relações entre o SA (classificações obtidas nos testes das disciplinas que constituem o currículo escolar), os padrões de AF (Physical Activity Questionnaire for Older Children) e os níveis de auto-estima (Physical Self-description Questionnaire) de 333 crianças de ambos os géneros dos 8 aos 12 anos de idade, os autores observaram que somente a auto-estima dos rapazes pareceu sofrer uma substancial influência positiva da AF praticada pelas crianças. Entretanto, em 2007, Sigfúsdóttir, Kristjánsson e Allegrante desenvolveram um trabalho de investigação com 5.810 alunos 
islandeses de ambos os géneros com 14 e 15 anos de idade e encontraram uma pequena mas significante associação positiva entre a frequência da $\mathrm{AF} e$ o grau de SA avaliado pelas classificações obtidas nas disciplinas nucleares do programa curricular (matemática e língua oficial local). Nos EUA, Cottrell, Northrup, e Wittberg (2007) estudaram a relação entre a pressão arterial enquanto factor de risco de doenças cardiovasculares (DCV), a ApM, avaliada através do cálculo do IMC, a ApF, medida pelo desempenho em cinco testes do FITNESSGRAM (resistência aeróbia, força abdominal, força superior, flexibilidade e força média), e o SA, obtido a partir dos resultados do West Virginia Educational Standards Test (WESTEST) composto por quatro áreas curriculares (matemática, leitura/escrita, ciências e estudos sociais), de 968 alunos do $5^{\circ}$ ano de escolaridade. Observaram em crianças consideradas obesas uma associação inversa e significativa com os resultados de todas as áreas, excepto em estudos sociais. Relativamente à relação entre os factores de risco de doenças cardiovasculares e o desempenho académico, os mesmo autores verificaram que crianças com valores mais elevados de tensão arterial obtiveram menor classificação em Ciências, comparativamente às crianças dentro dos valores normais de tensão arterial, não se registando diferenças significativas nas outras áreas curriculares. Do mesmo modo, crianças com menor pontuação nos testes de ApF alcançaram resultados inferiores em todas as áreas curriculares, comparativamente às que obtiveram níveis superiores.

Ainda nos EUA, em Seattle, Martin, e Chalmers (2007) investigaram a relação entre a ApF (President's Challenge) e o SA (lowa Tests of Basic Skills) numa amostra de 5847 alunos do $3^{\circ}, 4^{\circ}, 6^{\circ}$ e $8^{\circ}$ ano de ambos os géneros. $A$ correlação relatada entre os valores médios da ApF e SA foi positiva e estatisticamente significativa mas de baixo valor. No mesmo ano, Castelli, Hillman, Buck, e Erwin (2007), ao estudarem a relação entre a ApF (push-ups, curl-ups, hip flexor, hamstring), o IMC (ApM) e o SA (Illinois Standard Achievement Test) de 582 crianças do $3^{\circ}$ e $5^{\circ}$ anos de escolaridade de ambos os géneros, observaram uma correlação positiva entre a ApF e o SA, e uma correlação negativa entre a ApM e o SA dos participantes.

Mais recentemente, Chomitz et al. (2009) encontraram também uma relação positiva e significativa entre o SA em Matemática e Inglês (Massachusetts Comprehensive Assesment System) e a ApF de 1.847 
crianças e jovens norte-americanos dos $4^{\circ}, 6^{\circ}, 7^{\circ}$ e $8^{\circ}$ anos de escolaridade de ambos os géneros. A probabilidade de sucesso das crianças nos dois testes do MCAS aumentou à medida que aumentou a quantidade de componentes positivas nos testes de ApF.

\section{Considerações Finais}

Pela cronologia dos estudos, verifica-se uma tendência crescente para a concretização de investigações de grande dimensão amostral e em que os factores associados ao DM (com especial incidência para a AF) surgem em destaque ao lado das variáveis marcadoras do SA.

A análise comparativa dos resultados obtidos nos diferentes estudos revistos permite observar alguma heterogeneidade ao nível das relações encontradas entre os diferentes factores associados ao DM (AF, ApF, ApM e $\mathrm{ApC}$ ) e o Sucesso Académico de crianças e jovens. Na generalidade dos estudos são referidas associações positivas entre as variáveis, mesmo que em alguns casos o valor seja baixo e/ou não significativo.

Os estudos referenciados reforçam ainda a importância dos factores associados ao desenvolvimento motor (AF, ApF, ApM e ApC) para a manutenção da função cognitiva em adultos, mesmo que esta relação pareça não estar ainda consolidada em idades precoces (Trudeau \& Shephard, 2008). Relevante é também a conclusão de que a prática de actividade física parece originar o desenvolvimento de capacidades e atitudes precursoras de um bom desempenho ao longo do percurso escolar (e.g. concentração, capacidade de aprendizagem, comportamentos assertivos em sala de aula, auto-estima, auto-imagem, etc.).

No seu conjunto, estas conclusões são de extraordinária relevância no que ao desenvolvimento integral, holístico da criança diz respeito. Repare-se no reconhecimento generalizado de que crianças com mais êxito nas tarefas motoras, que evidenciam melhor robustez muscúlo-atlética, e que passam mais tempo em actividades de movimento, apresentam um sucesso académico melhorado ou, no limite, igual ao das outras crianças. Como as vantagens da prática da actividade física, e de melhor aptidão física, motora, e morfológica, são inequívocas para um desenvolvimento saudável, todos estes conjuntos de estudos e resultados são peremptórios em confirmar que uma mente sã se pode fazer acompanhar idealmente de um corpo são. 
A mensagem aqui deixada é claríssima e sempre demonstrada na sua essência pelos resultados dos estudos de intervenção e longitudinais: a sociedade, o sistema educativo, os professores e os pais devem investir sem medo na promoção dos tempos e oportunidades de estimulação motora das suas crianças, alunos e filhos. Especificamente, o sistema educativo deve continuar a promover a articulação estruturada dos tempos de Educação Física curricular e dos momentos activos de lazer e tempo livre (não curriculares). Só assim poderemos ter gerações de adultos mais bem preparados para enfrentar os desafios (físicos e intelectuais) do mundo de amanhã. Só assim estaremos na Escola e na família a promover efectivamente um desenvolvimento saudável, harmonioso e completo das nossas crianças. Só assim estaremos a promover o verdadeiro sucesso escolar das gerações vindouras.

\section{Referências}

Ahamed, Y., Macdonald, H., Reed, K., Naylor, P. J., Lui-Ambrose, T., \& McKay, H. (2007). School-based physical activity does not compromise children's academic performance. Med Sci Sports Exerc, 39, 371-376.

Andersen, L., Hasseltrom, H., Gronfeldt, V., Hansen, S., \& Karsten, K. (2004). The relationship between physical fitness and clustered risk, and tracking of clustered risk from adolescence to young adulthood, eight years follow-up in the Danish Youth and Sport Study. International Journal of Behavioral Nutrition and Physical Activity, 1 (6). doi:10.1186/1479-5868-1-6.

Blair, S., \& Church, T. (2004). The fitness, obesity, and health equation. Is physical activity the common denominator? JAMA, 292, 1232-1234.

Burton, L., \& VanHeest, J. (2007). The Importance of Physical Activity in Closing the Achievement Gap. Quest, 59, 212-218.

Carlson, S. A., Fulton, J. E., Lee, S. M., Maynard, L. M., Brown, D. R., et al. (2008). Physical Education and Academic Achievement in Elementary School: Data From the Early Childhood Longitudinal Sutdy. American Journal of Public Health, 98, 721-727.

Castelli, D. M., Hillman, C. H., Buck, S. M., \& Erwin, H. E. (2007). Physical Fitness and Academic Achievement in Third- and Fifth-Grade Students. Journal of Sport \& Exercise Psychology, 29, 239-252.

Chomitz, V., Slining, M., McGowan, R., Mitchell, S., Dawson, G., \& Hacker, K. (2009). Is there a relationship between physical fitness and academic achievement? Positive results from public school children in the northeastern United States. $J$ Sch Health, 79, 30-37.

Coe, D. P., Pivarnik, J. M., Womack, C. J., Reeves, M. J., \& Malina, R. M. (2006). Effect of physical education and activity levels on academic achievement in children. Med Sci Sports Exerc, 38, 1515-1519. 
Cottrell, L. A., Northrup, K., \& Wittberg, R. (2007). The Extended Relationship between Child Cardiovascular Risks and Academic Performance Measures. Obesity, 15, 3170-3177.

Dwyer, T., Coonan, W., Leitch, D., Hetzel, B., \& Baghurst, R. (1983). An investigation of the effects of daily physical activity on the health of primary school students. Int J Epidemiol, 12, 308-313.

Dwyer, T., Sallis, J., Blizzard, L., Lazarus, R., \& Dean, K. (2001). Relation of academic performance to physical activity and fitness in children. Pediatr Exerc Sci, 13, 225-338.

Grissom, J. (2005). Physical fitness and academic achievement. California Department of Education. JEP online, 8, 11-25.

Hillman, C., Castelli, D., \& Buck, S. (2005). Aerobic fitness and neurocognitive function in healthy preadolescent children. Med Sci Sports Exerc, 37, 1967-1974.

Kim, H-Y., Frongillo, E., Han, S-S., Oh, S-Y., Kim W-K. et al. (2003). Academic performance of Korean children is associated with dietary behaviours and physical status. Asia Pacific J Clin Nutr, 12, 186-192.

Martin, L., \& Chalmers, G. (2007). The relationship between academic achievement and physical fitness. Physical Educator, 64, 214-221.

Mo-Suwan, L., Lebel, L., Puetpaiboon, A., \& Junjana, C. (1999). School performance and weight status of children and young adolescents in a transitional society in Thailand. International Journal of Obesity, 23, 272-277.

Nelson, M., \& Gordon-Larsen, P. (2006). Physical activity and sedentary behavior patterns are associated with selected adolescent health risk behaviors. Pediatrics, 117, 1281-1290.

Pate, R., Heath, G., Dowda, M., \& Trost, S. (1996). Associations between Physical Activity and Other Health Behaviors in a Representative Sample of US Adolescents. American Journal of Public Health, 86, 1577-1581.

Raviv, S., Reches, I., \& Hecht, O. (1994). Effects of activities in the motor-cognitivelearning center on academic achievements, psychomotor and emocional development of children (aged 5-7). J Phys Educ Sport Sci (Israel), 2, 50-84.

Sallis, J., McKenzie, T., Kolody, B., Lewis, M., Marshall, S., \& Rosengard, P. (1999). Effects of health-related physical education on academic achievement: Project SPARK. Research Quarterly for Exercise and Sport, 70, 127-134.

Shephard, R., Volle, M., Lavallée, H., LaBarre, R., Jéquier, J., \& Rajic, M. (1984). Required physical activity and academic grades: a controlled logitudinal study. In J. Ilmarinen \& J. Valimaki (Org.), Children and Sport (pp 58-63). Berlin: Springer Verlag.

Sibley, B., \& Etnier, J. (2003). The relationship between physical activity and cognition in children: A meta-analysis. Pediatric Exercise Science, 15, 243-256.

Sigfúsdóttir, I., Kristjánsson, A., \& Allegrante, J. (2007). Health behaviour and academic achievement in Icelandic school children. Health Educ Res, 22, 70-80.

Taras, H. (2005). Physical Activity and Student Performance at School. Journal of School Health, 75, 214-218. 
Tomporowski, P., Davis, C., Miller, P., \& Naglieri, J. (2008). Exercise and Children's Inteligence, Cognition and Academic Achievement. Educ Psychol Rev, 20, 111131.

Trudeau, F., \& Shephard, R. (2008). Physical education, school physical activity, school sports and academic performance. International Journal of Behavioral Nutrition and Physical Activity, 5, 10. doi:10.1186/1479- 5868-5-10.

Wassenberg, R., Feron, F., Kessels, A., Hendriksen, J., Kalff, A., \& Kroes, M. (2005). Relation between Cognitive and Motor Performance in 5- to 6-Year-Old Children: Results from a Large-Scale Cross-Sectional Study. Child Development, 76, 1092-1103.

Willms, J. (2003). Ten Hypotheses about Socioeconomic Gradients and Community Differences in Children's Developmental Outcomes (final report). Otawa: Human Resources Development Canada. Disponível em http://www.unb.ca/crisp/pdf/0305.pdf

Yu, C., Chan, S., Cheng, F., Sung, R., \& Hau, K. (2006). Are physical activity and academic performance compatible? Academic achievement, conduct, physical activity and self-esteem of Hong Kong Chinese primary school children. Educational Studies, 32, 331-341.

Who (1999). Health 21 - Health for all in the 21st Century. Copenhagen: WHO Europe. 


\section{MOTOR DEVELOPMENT AND ACADEMIC ACHIEVEMENT. IS THERE A} RELATIONSHIP IN CHILDREN AND YOUNGSTERS?

\section{Abstract}

A holistic perspective of the human development needs to look for every element responsible for the individual success. Surely between those, motor development for its relation with healthy lifestyles, and academic achievement due to its social relevance, constitute two major determinants of future success in life. The aim to this article was to review the major contributions found in the scientific literature of the last decade that can help us to better understand the possible relationship between motor development success and academic achievement in children and adolescents.

Keywords

Motor development; Academic achievement; Children; Adolescents

DÉVELOPPEMENT MOTEUR ET SUCCÈS ACADÉMIQUE. EST CE QU'IL Y A UNE RELATION ENTRE DES ENFANTS ET DES JEUNES?

\section{Résumé}

L'adoption d'une perspective holistique du développement nous oblige à revoir la relation entre les éléments du développement personnel en herbe. Nous détacherons les plus saillants: 1) le succès du développement moteur des enfants et des jeunes, en rapport à la santé et à la définition de modes de vie d'épanouissement; 2) la réussite académique, par sa valorisation sociale, par l'importance de la détermination personnelle au niveau des choix de carrière à venir, et par sa présence constante aux moments qui précèdent la vie adulte. Le présent travail constitue une révision de la littérature sur les relations existantes entre les facteurs associés au développement moteur - 
notamment l'aptitude physique, morphologique, coordinatrice et l'activité physique - et la réussite académique des enfants et des jeunes, revisitée sous une perspective holistique.

Mots-clé

Succès académique; Développement moteur; Enfants; Jeunes

Recebido em Setembro/2009 Aceite para publicação em Outubro/2010

Toda a correspondência relativa a este artigo deve ser enviada para: Luís Paulo Rodrigues, Escola Superior Educação Viana do Castelo, Av. Capitão Gaspar de Castro, Apartado 513, 4901-908 Viana do Castelo, Portugal. Telefone 258806200, Telefax 258806209, E-mail: Iprodrigues@ese.ipvc.pt 